March 2008;131:747-759). (Respond: Hanns Lochmuller MD, Institute of Human Genetics, University of Newcastle upon Tyne, International Centre for Life, Central Parkway, Newcastle upon Tyne NE1 3BZ, UK).

COMMENT. Molecular genetic testing is important in diagnosis and therapy of infants with CMS. The administration of esterase inhibitors in patients with COLQ mutations can result in serious complications, and an initial short-term beneficial effect may be misleading. Ephedrine ( 2 to $3 \mathrm{mg} / \mathrm{kg} /$ day) was the most effective therapy in the above study. Most patients with COLQ mutations are disabled from infancy, and muscle weakness is progressive and complicated by ventilatory insufficiency and scoliosis. Clinical diagnosis is supported by repetitive CMAP, and increased muscle weakness following administration of pyridostigmine. COLQ gene mutations are the third most common cause of CMS, and occur as frequently as DOK7 mutation cases.

\title{
HISTOCHEMICAL ABNORMALITIES IN VARIOUS FORMS OF CONGENITAL MUSCULAR DYSTROPHY
}

A large Australasian cohort of patients with congenital muscular dystrophy (CMD) was screened to determine the frequency of various forms, in a study at Children's Hospital at Westmead; the University of Sydney; University of Melbourne, Australia; and University of Nevada, Reno; and University of Illinois, Chicago. Of 101 patients, $45 \%$ were screened by immunofluorescence and showed abnormal staining for glycosylated-a-dystroglycan (DG) in $25 \%$. Half of these had reduced DG by Western Blot test. All patients with abnormal DG staining had DNA sequencing of the fukutin-related protein, fukutin, POMGnT1 and POMT1 genes, and mutations were identified in one patient for each of the genes. Abnormalities in collagen VI immunofluorescence were identified in $12 \%$ of CMD patients, COL6 mutations in 8 of 9 patients tested, and laminin a-2 deficiency occurred in $8 \%$ of cases. (Peat RA, Smith JM, Compton AG et al. The diagnosis and etiology of congenital muscular dystrophy. Neurology Dec 26, 2007 (Epub ahead of print)).

COMMENT. Various histochemical and DNA sequencing abnormalities are identified in a large cohort of CMD cases. Patients with abnormal glycosylated adystroglycan immunofluorescence were most common in this cohort. Other studies have suggested that the cause of $50 \%$ of all CMDs is a primary deficiency in laminin a2, and Ullrich CMD is a second most common form. Molecular diagnostic testing is important for genetic counseling and an emerging new era of gene therapy (Rando TA. Get personal with gene therapy for muscular dystrophy. Lancet Neurology 2008;7:196-8).

\section{MRI IN CONGENITAL FACIAL PALSY}

Magnetic resonance (MR) findings in a 12-month-old boy with congenital unilateral facial palsy and a 9-month-old girl with atypical Moebius syndrome are reported from the National Center of Neurology and Psychiatry, Kodaira, Japan. In the boy with unilateral palsy, MRI showed an asymmetry of internal auditory channels with absence of the right facial nerve. MRI on the girl with Moebius syndrome showed a slightly hypoplastic 